\title{
Particulate matter 2.5 regulates lipid synthesis and inflammatory cytokine production in human SZ95 sebocytes
}

\author{
QIN LIU ${ }^{1}$, JIANBO WU ${ }^{2}$, JIQUAN SONG $^{2}$, PIN LIANG $^{2}$, KAIPING ZHENG $^{2}$, GUIFENG XIAO $^{2}$, \\ LANTING LIU ${ }^{2}$, CHRISTOS C. ZOUBOULIS ${ }^{3}$ and TIECHI LEI ${ }^{1}$ \\ ${ }^{1}$ Department of Dermatology, Renmin Hospital of Wuhan University, Wuchang, Wuhan, Hubei 430060; \\ ${ }^{2}$ Department of Dermatology, Zhongnan Hospital of Wuhan University, Wuchang, Wuhan, Hubei 430071, P.R. China; \\ ${ }^{3}$ Department of Dermatology, Venereology, Allergology and Immunology, Dessau Medical Center, \\ Theodore Fontane Medical University of Brandenburg, Dessau D-06847, Germany
}

Received May 15, 2017; Accepted August 21, 2017

DOI: 10.3892/ijmm.2017.3109

\begin{abstract}
A large body of evidence indicates that particulate matter (PM)2.5 is associated with various negative effects on human health. However, the impact and molecular mechanism of PM2.5 on the skin have not been elucidated. Therefore, the present study aimed to investigate the effects of two types of PM2.5 [water-soluble extracts (W-PM2.5) and non-water-soluble extracts (NW-PM2.5)] on cell proliferation, cell cycle progression, lipid synthesis, and inflammatory cytokine production of human SZ95 sebocytes. The results demonstrated that NW-PM2.5 and W-PM2.5 exposure dose-dependently inhibited SZ95 sebocyte proliferation by inducing $G_{1}$ cell arrest. Furthermore, NW-PM2.5 and W-PM2.5 significantly reduced sebaceous lipid synthesis and markedly promoted the production of inflammatory cytokines, including interleukin-1 $\alpha$ (IL-1 $\alpha$ ), IL-6 and IL-8 in SZ95 sebocytes. Additionally, the expression of aryl hydrocarbon (Ah) receptor (AhR), AhR nuclear translocator protein (ARNT), as well as cytochrome P450 1A1 were significantly increased following PM2.5 exposure. Thus, these findings indicate that PM2.5 exerts inhibitory effects on cell proliferation and lipid synthesis, and stimulatory effects on inflammatory cytokine production and AhR signaling activation in human SZ95 sebocytes.
\end{abstract}

\section{Introduction}

Increasing evidence has demonstrated the association between air pollution and sebaceous gland-associated diseases (1-4). Recently, studies regarding environmental pollutants indicated that dioxins inhibit lipid synthesis and induce differentiation

Correspondence to: Dr Tiechi Lei, Department of Dermatology, Renmin Hospital of Wuhan University, 99 ZhangZhiDong Road, Wuchang, Wuhan, Hubei 430060, P.R. China

E-mail: tchlei@whu.edu.cn

Key words: particulate matter 2.5, lipid synthesis, inflammatory cytokines, aryl hydrocarbon receptor signaling, cytochrome P450 1A1 of human sebaceous cells into keratinocytes, leading to the development of clinical chlorine acne (5). Hu et al (6) also identified that cigarette smoke extract benzo(a)pyrene regulates the synthesis of lipids and induce pro-inflammatory effects in human sebocytes, as evidenced by promoting the release of pro-inflammatory cytokines [interleukin-1 $\alpha$ (IL- $1 \alpha)$, IL-6 and IL-8] (6). The above-mentioned studies demonstrate that environmental pollution is closely associated with the synthesis and secretion of sebaceous glands, and potentially involved in the development of sebaceous gland-associated diseases (7).

Particulate matter (PM)2.5, also termed fine particles, were identified as PM, which are $<2.5 \mu \mathrm{m}$ in diameter (8). Due to the small particle size and specific surface area, PM2.5 easily penetrate the alveoli or even the blood, which increases the risk of human health problems $(9,10)$. A recent study demonstrated that ambient PM2.5 increases the risk of eczema and other skin diseases (11). However, to the best of our knowledge, the effects of PM2.5 on the function of human sebaceous glands have not yet been elucidated.

The key molecular signaling pathway involved in the response of skin cells against environment pollutants is the aromatic hydrocarbon receptor (AhR) pathway $(12,13)$. AhR, also termed dioxin receptor, is a ligand-activated transcription factor expressed in all skin cells, including keratinocytes (14), fibroblasts (15) and SZ95 sebocytes cells (16) in vitro and in human skin in vivo (17). The formation of the AhR/AhR nuclear translocator (ARNT) heterodimer activates the cytoplasmic cytochrome P4501 A1 (CYP1A1) $(18,19)$. Furthermore, increasing evidence has highlighted that $\mathrm{AhR}$ is significant in cell growth, proliferation, cell cycle progression (20), cell differentiation and inflammatory responses (21) in the absence of external ligands. Recently, Kakimoto et al (22) identified that PM2.5 has the highest AhR ligand activity among all of the particle sizes, which supports the possible involvement of AhR signaling in the mechanism of PM2.5-induced skin diseases. However, whether the PM2.5 effects on SZ95 sebocytes are associated with AhR/CYP1A1 signaling remains unclear.

Therefore, the present study aimed to investigate the influences of PM2.5 on human SZ95 sebocytes, and to investigate the relevant mechanisms. The effects of 
water-soluble extract (W-PM2.5) and non-water-soluble extract (NW-PM2.5) exposure on cell proliferation, cell cycle progression, lipid synthesis and the inflammatory response were investigated in human SZ95 sebocytes. Furthermore, whether the AhR/CYP1A1 signaling pathway is involved in these effects was investigated.

\section{Materials and methods}

Collection and extraction of PM2.5. PM2.5 was collected by TH-150C Medium flow air total suspended particulate sampler (Wuhan TianHong Environmental Protection Industry Co., Ltd., Wuhan, China) (100 1/min) in Wuhan urban air during July 2015. The sample was set on the rooftop of a building of the Institute of Atmospheric Research, School of Environmental Studies, China University of Geosciences (Wuhan, China), $\sim 6 \mathrm{~m}$ above the ground. The filter was changed every $24 \mathrm{~h}$ using the air total particle sampler. Subsequent to sampling, the PM2.5 filter was placed under an ultraviolet lamp for $30 \mathrm{~min}$ and cut into two equal size pieces. The W-PM2.5 and NW-PM2.5 were isolated and prepared as previously described (23). The samples were collected and stored at $-80^{\circ} \mathrm{C}$ until later use.

Cell culture and exposure. Immortalized human SZ95 sebaceous gland cells, (patented cell, gifted by Professor Christos C. Zouboulis, Department of Dermatology, Venereology, Allergology and Immunology, Dessau Medical Center, Theodore Fontane Medical University of Brandenburg, Germany) at passages 30-40 were cultured at $37^{\circ} \mathrm{C}$ in Sebomed ${ }^{\circledR}$ basal medium (Biochrom $\mathrm{GmbH}$, Berlin, Germany) supplemented with $10 \%$ fetal calf serum, Gibco $5 \mathrm{ng} / \mathrm{ml}$ human epidermal growth factor (Thermo Fisher Scientific, Inc., Waltham, MA, USA), $100 \mathrm{U} / \mathrm{ml}$ penicillin and $100 \mathrm{~g} / \mathrm{ml}$ streptomycin (Gibco; Thermo Fisher Scientific, Inc.). Various concentrations (1, 10, 100 and $250 \mu \mathrm{g} / \mathrm{ml}$ ) of PM2.5 were prepared. For the exposure, cells were exposed to a W-PM2.5 or NW-PM2.5 for $48 \mathrm{~h}$.

Cell counting kit-8(CCK-8) assay. Following exposure for $48 \mathrm{~h}$, cell viability was determined using CCK-8 assay kits (C0038; Shanghai Beyotime Biotechnology Co., Ltd., Shanghai, China) according to the manufacturer's instructions. CCK-8 solution $(10 \mu \mathrm{l})$ was added to each well, followed by an incubation for 1-4 h. Absorbance was measured using a microplate reader at a wavelength of $450 \mathrm{~nm}$. The cells treated with the solvent served as a control group and wells without cells served as the blank group. Cell viability was calculated according to the following formula: cell proliferation $(\%)=$ optical density $(\mathrm{OD})$ of the experimental group/OD of the control group $\mathrm{x} 100$.

Cellular toxicity. SZ95 sebocytes were seeded in 96-well plates. Subsequent to exposure for $48 \mathrm{~h}$, lactate dehydrogenase (LDH) activity was detected using a Pierce LDH cytotoxicity assay kit (Thermo Fisher Scientific, Inc.) according to the manufacturer's protocol.

Cell cycle assay. SZ95 sebocytes were seeded into 6-well plates. Following exposure for $48 \mathrm{~h}$ with different concentrations of PM2.5 suspension, cells were collected using $0.25 \%$ trypsin and phosphate-buffered saline (PBS). Also, culture was centrifuged at $300 \mathrm{x} \mathrm{g}$ for $5 \mathrm{~min}$ at room temperature twice. After that precooled $90 \%$ ethanol was added. Then cells were resuspended at $4^{\circ} \mathrm{C}$ for $20 \mathrm{~min}$ and centrifuged at $300 \mathrm{x} \mathrm{g}$ for $5 \mathrm{~min}$ at room temperature. Cells were incubated with propidium iodide (PI) buffer $[50 \mathrm{mg} / \mathrm{ml}$ containing ribonuclease $\mathrm{A}(50 \mathrm{ng} / \mathrm{ml})]$ at room temperature and stained in the dark for $20 \mathrm{~min}$ at room temperature. Cell cycle distribution was analyzed using a BD Biosciences Flow Cytometer (BD Biosciences, San Jose, CA, USA).

Oil Red O staining. SZ95 sebocytes were seeded in 24-well plates $\left(5 \times 10^{4}\right.$ cells/well). After a 48 -h exposure with different concentrations of PM2.5 suspension, cells were washed with PBS and fixed in $10 \%$ neutral formaldehyde for $30 \mathrm{~min}$. The cells were then washed twice in distilled water for 2 min, stained with Oil Red O dye (AS1083; Wuhan Aspen Biotechnology Co., Ltd., Wuhan, China) (0.5\% Oil Red dissolved in isopropanol, then diluted with distilled water at a ratio of 6:4) at room temperature in the dark for $10 \mathrm{~min}$ and washed in distilled water for $1 \mathrm{~min}$. Subsequently, cells were counterstained with hematoxylin, and then sealed by glycerol gelatin for long-term preservation, and observed under an inverted Olympus microscope.

Enzyme-linked immunosorbent assay (ELISA). SZ95 sebocytes were seeded in 24 -well plates $\left(1 \times 10^{5}\right.$ cells/well). After 48-h exposure to different concentrations of PM2.5 suspension, the supernatant was collected at $4^{\circ} \mathrm{C}$, centrifuged for $20 \mathrm{~min}$ at $1,000 \mathrm{xg}$ at $4^{\circ} \mathrm{C}$, and the supernatant was collected and stored at $-20^{\circ} \mathrm{C}$ for subsequent assays. Concentrations of IL-1 $\alpha$, IL- 6 and IL- 8 were determined using commercial ELISA kits (E-EL-H0088c, E-EL-H0102c and E-EL-H0048c; ElabScience Biotechnology Co., Ltd., Wuhan, China) according to the manufacturer's instructions.

Immunofluorescentcytochemical analysis. Immunofluorescent cytochemical analysis was performed using anti-AhR (17840-1-AP), anti-ARNT (14105-1-AP), anti-CYP1A1 (13241-1-AP) (all from Proteintech Group, Inc., Wuhan, China) and Cy3-conjugated anti-rabbit IgG antibodies (AS-1109; Wuhan Aspen Biotechnology Co., Ltd.). SZ95 sebocytes were seeded into 6-well plates. Following exposure for $48 \mathrm{~h}$ to various concentrations of PM2.5 suspension, coverslips were added to the plates and incubated with $5 \% \mathrm{CO}_{2}$ at $37^{\circ} \mathrm{C}$ until cells reached $80 \%$ confluence. Media was aspirated from the plates and washed 3 times with PBS. Cells were fixed with 4\% paraformaldehyde for $30 \mathrm{~min}$ at room temperature. We then added Triton X-100 for 10 min and hydrogen peroxide solution at room temperature in the dark for $20 \mathrm{~min}$; after each step, the cells were washed 3 times with PBS for $5 \mathrm{~min}$. The cell culture was incubated with primary antibody overnight at $4^{\circ} \mathrm{C}$ and washed 3 times for 5 min with PBS. Stained with conjugated secondary antibody for $30 \mathrm{~min}$ at room temperature for $50 \mathrm{~min}$ and washed 3 times for $5 \mathrm{~min}$ with PBS. Nuclear staining was then performed with DAPI. Images of each slide were obtained using an optical microscope system (Axiomager; Carl Zeiss AG, Oberkochen, Germany). The protein expression levels of AhR, ARNT and CYP1A1 were quantified with an image analysis program (Q-imaging Pro-7; Olympus, Tokyo, Japan) from the microscope system. 
A

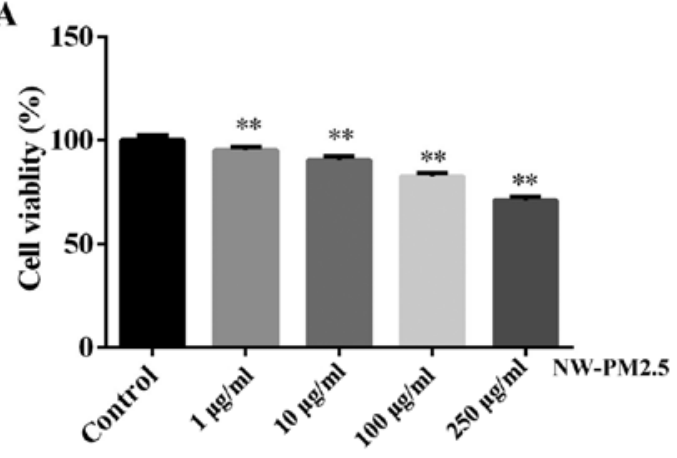

C

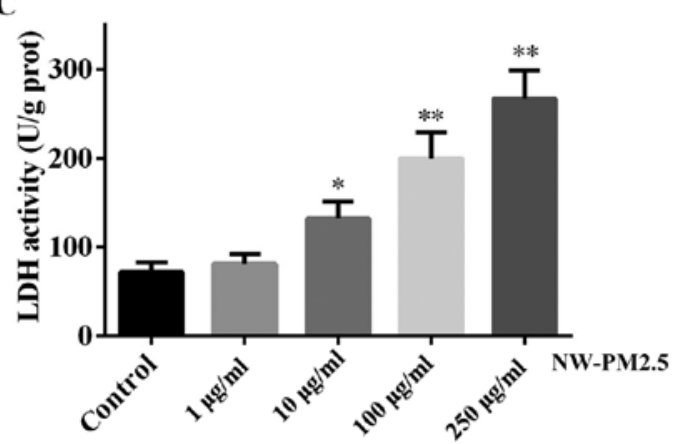

B

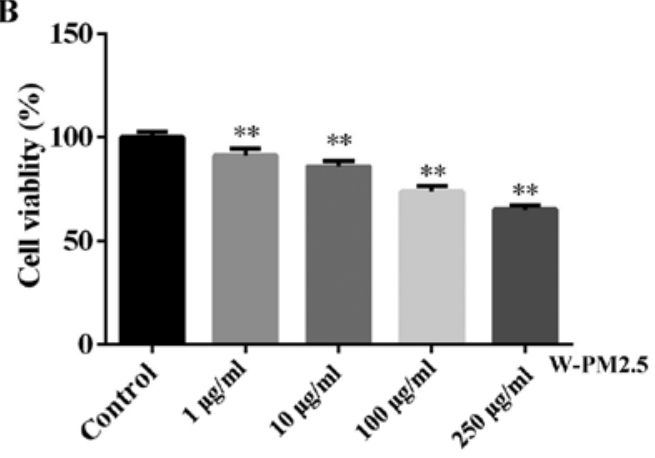

D

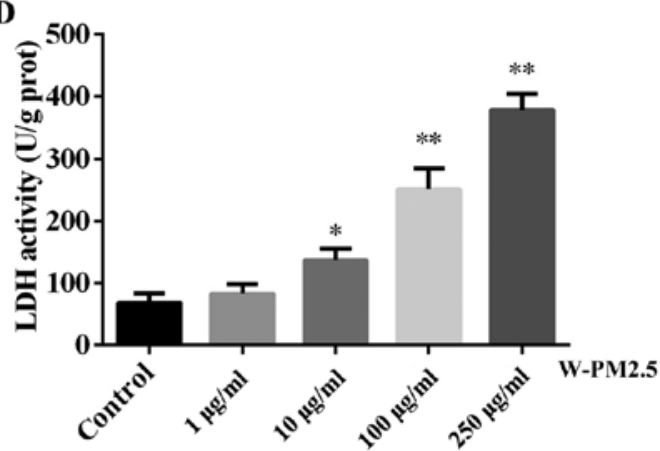

Figure 1. Cytotoxic effect of PM2.5 on SZ95 sebocytes. Following treatment of SZ95 sebocytes with (A) NW-PM2.5 and (B) W-PM2.5 for 48 h, cell viability was measured by cell counting kit-8 assay and LDH activity in (C) NW-PM2.5 and (D) W-PM2.5 cells. Data are expressed as means \pm standard error of the mean $(n=3),{ }^{*} \mathrm{P}<0.05$ and ${ }^{* *} \mathrm{P}<0.01$ vs. control. NW-PM2.5, non-water-soluble extracts; W-PM2.5, water-soluble extracts; PM, particulate matter; LDH, lactate dehydrogenase.

Western blotting. Following exposure for $48 \mathrm{~h}$ to various concentrations of PM2.5 suspension ,the adherent cells were washed with TBS buffer for 3 times. After drying the residual liquid, protease inhibitor was added for $3 \mathrm{~min}$ at room temperature. The cell total protein extraction reagent was then added for $5 \mathrm{~min}$ at room temperature. Cells were collected to $1.5 \mathrm{ml}$ centrifuge tubes and centrifuged at $10,000 \times \mathrm{g}$ for $5 \mathrm{~min}$ at $4^{\circ} \mathrm{C}$. The supernatant which was the total protein solution was collected at $4^{\circ} \mathrm{C}$. The concentration of the proteins was determined using a BCA protein assay kit (AS1086; Wuhan Aspen Biotechnology Co., Ltd.). Total protein samples (ensure that the total protein amount of each sample was $40 \mu \mathrm{g}$ ) were separated with $10 \%$ sodium dodecyl sulfate-polyacrylamide gel electrophoresis (SDS-PAGE), then transferred onto polyvinylidene fluoride membrane (EMD Millipore, Bedford, MA, USA), blocked with skimmed milk and incubated overnight at $4{ }^{\circ} \mathrm{C}$ with primary antibodies against anti-AhR (dilution, 1:1,000), anti-ARNT (dilution, 1:500), CYP1A1 (dilution, 1:500) and glyceraldehyde 3-phosphate dehydrogenase (GAPDH) (dilution, 1:1,000). The membranes were incubated with the secondary antibody (horseradish peroxidase goat anti rabbit; dilution, 1:10,000) for $30 \mathrm{~min}$ at room temperature, and the blots were detected using an enhanced chemiluminescence kit (Cell Signaling Technology, Inc., Danvers, MA, USA). GAPDH served as an internal control.

Statistical analysis. Results are expressed as the mean \pm standard error of the mean. Statistical analysis was performed using two-way ANOVA and Dunnett's post hoc test. $\mathrm{P}<0.05$ was considered to indicate a statistically significant difference.

\section{Results}

PM2.5 exposure inhibits the viability of SZ95 sebocytes. To evaluate the effects of PM2.5 exposure on the viability of SZ95 sebocytes, a CCK-8 assay was performed in the present study and the results are presented in Fig. 1. PM2.5 (NW-PM2.5 and W-PM2.5) exposure for $48 \mathrm{~h}$ significantly induced cell viability when compared with the control cells $(\mathrm{P}<0.01)$ (Fig. 1A and $\mathrm{B})$. No significant difference was identified between the viability of NW-PM2.5- and W-PM2.5-exposed SZ95 sebocytes $(\mathrm{P}>0.05)$. In addition, the toxicity of NW-PM2.5 and W-PM2.5 exposure on SZ95 sebocytes was evaluated using a Pierce LDH cytotoxicity assay kit according to the manufacturer's instructions. Compared with the control cells, NW-PM2.5 exposure $(10 \mu \mathrm{g} / \mathrm{ml}, \mathrm{P}<0.05 ; 100$ and $250 \mu \mathrm{g} / \mathrm{ml}, \mathrm{P}<0.01)$ significantly increased LDH activity in a dose-dependent manner (Fig. 1C). W-PM2.5 exhibited similar effects on SZ95 sebocytes (Fig. 1D).

PM2.5 exposure arrested the cell cycle at the $G_{1}$ phase in SZ95 sebocytes. The effects of PM2.5 on the cell cycle were investigated (Fig. 2). Flow cytometry demonstrated that compared with the unexposed control cells, the number of cells in the $G_{1}$ phase were markedly increased in cells exposed to NW-PM2.5 (Fig. 2A). With increasing NW-PM2.5 concentrations, the cell rates in the $G_{1}$ phase also significantly 

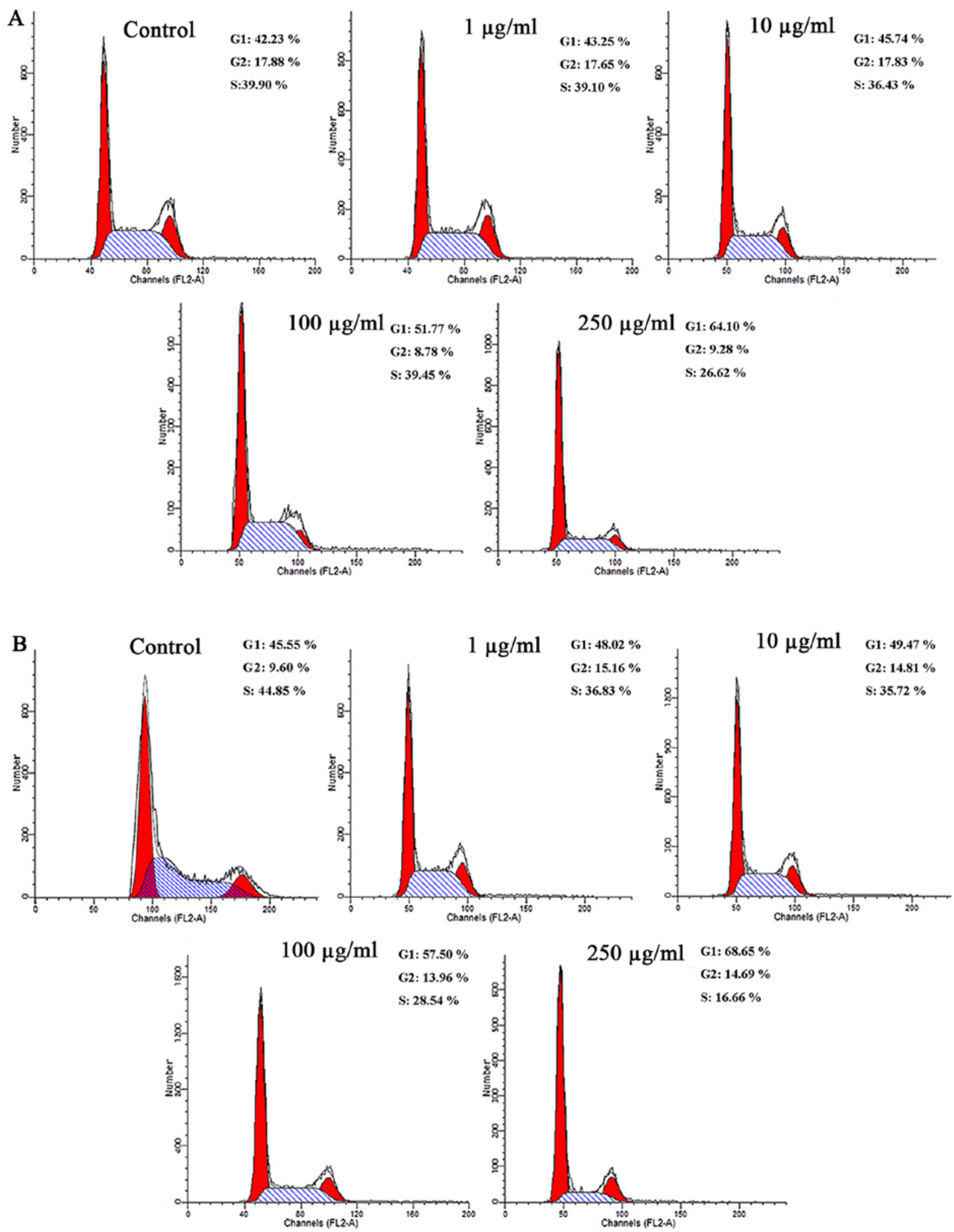

Figure 2. Effect of PM2.5 on cell cycle process of SZ95 sebocytes. Following treatment of SZ95 sebocytes with (A) NW-PM2.5 and (B) W-PM2.5 for 48 h, the cell cycle process was assessed by flow cytometry. NW-PM2.5, non-water-soluble extracts; W-PM2.5, water-soluble extracts; PM, particulate matter.

increased. As illustrated in Fig. 2B, compared with the control cells, W-PM2.5 exposure markedly increased the number of cells in the $\mathrm{G}_{1}$ phase in a dose-dependent manner.

PM2.5 exposure regulated lipid synthesis in SZ95 sebocytes. As lipids secreted by sebaceous gland cells are important in the process of skin physiology involving cell structure, cohesion and desquamation as well as formation and function of a permeability barrier, the effects of PM2.5 exposure on lipid synthesis were examined in $\mathrm{SZ95}$ sebocytes. Oil Red O staining indicated that NW-PM2.5 (Fig. 3A) and W-PM2.5 (Fig. 3B) at low concentrations promoted lipid synthesis, while high concentrations of NW-PM2.5 and W-PM2.5 markedly inhibited lipid synthesis in SZ95 sebocytes. 
A

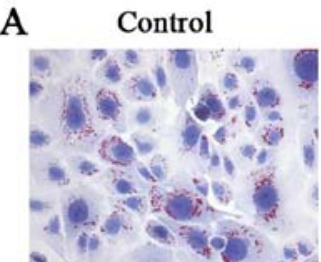

B

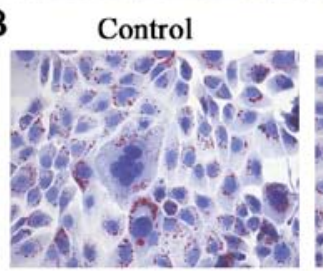

$1 \mu \mathrm{g} / \mathrm{ml}$

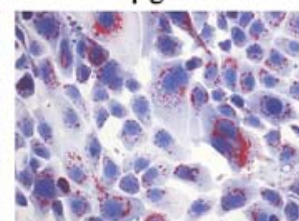

$1 \mu \mathrm{g} / \mathrm{ml}$

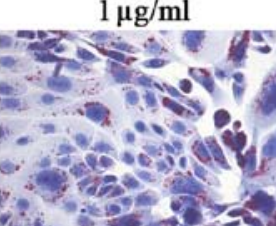

$10 \mu \mathrm{g} / \mathrm{ml}$

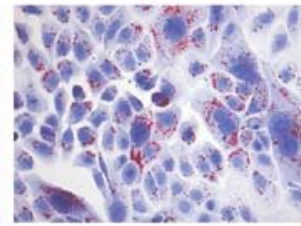

$10 \mu \mathrm{g} / \mathrm{ml}$

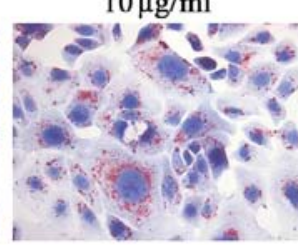

$100 \mu \mathrm{g} / \mathrm{ml}$

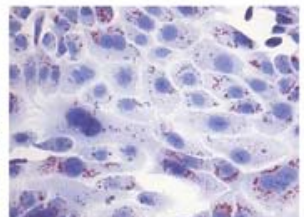

$100 \mu \mathrm{g} / \mathrm{ml}$

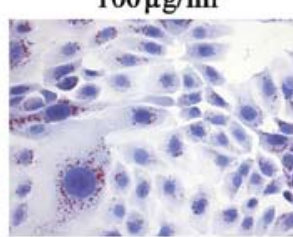

$250 \mu \mathrm{g} / \mathrm{ml}$

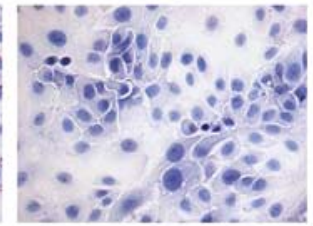

$250 \mu \mathrm{g} / \mathrm{ml}$

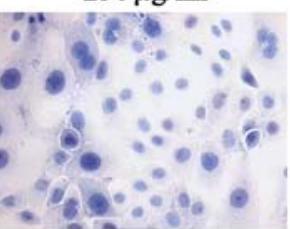

Figure 3. Lipid synthesis of SZ95 sebocytes following PM2.5 exposure. The intracellular lipids in (A) NW-PM2.5- and (B) W-PM2.5-exposed SZ95 sebocytes were visualized by Oil Red O staining. The magnification was x200. PM, particulate matter; NW-PM2.5, non-water-soluble extracts; W-PM2.5, water-soluble extracts.
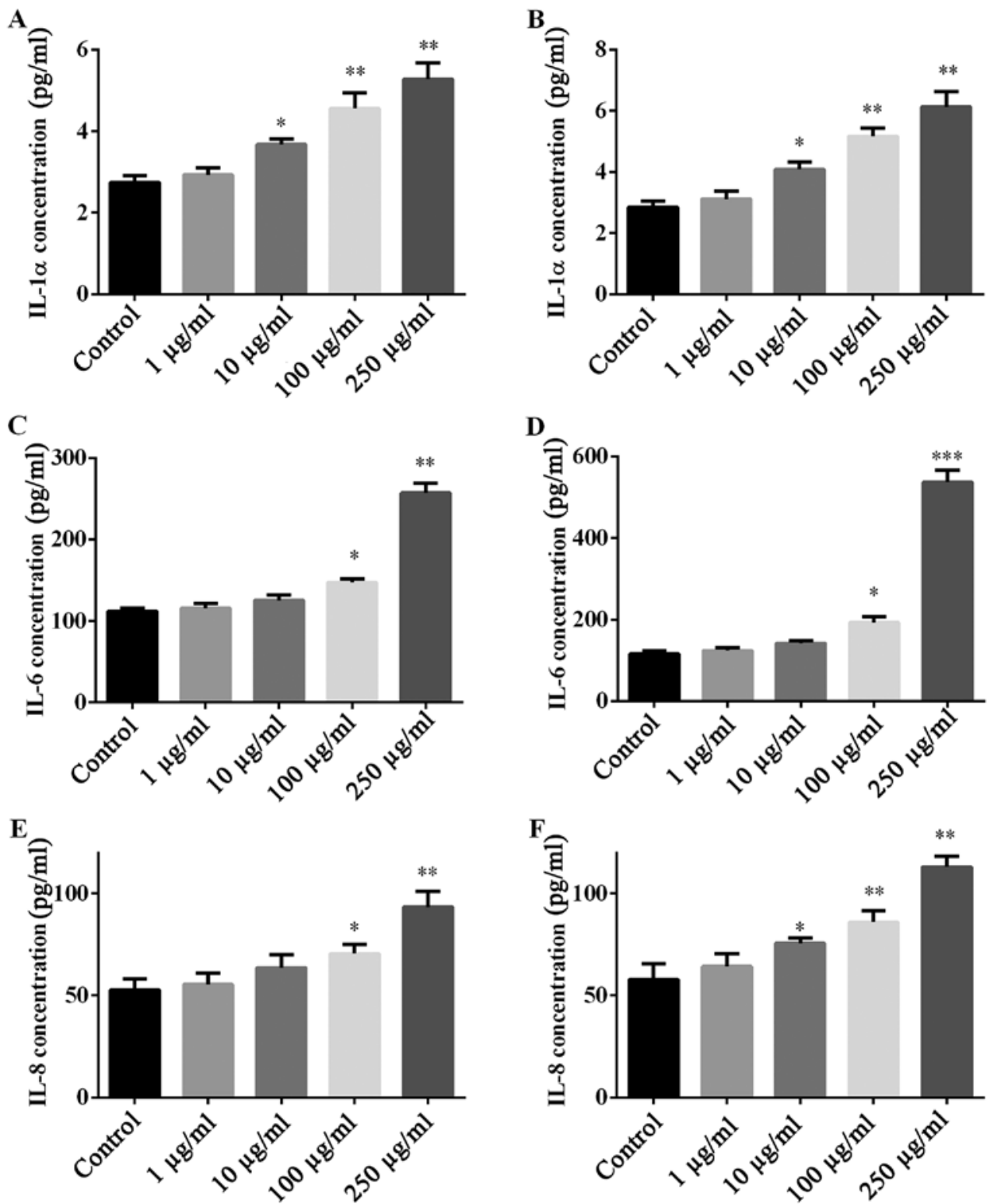

Figure 4. Secretion of IL-1 $\alpha$,IL-6 and IL-8 in SZ95 sebocytes following PM2.5 exposure. Subsequent to treatment of SZ95 sebocytes with (A,C and E) NW-PM2.5 and (B, D and F) W-PM2.5, the production of pro-inflammatory cytokines, IL-1 $\alpha$, IL- 6 and IL-8 in culture supernatant were measured by enzyme-linked immunosorbent assay. Data are expressed as means \pm standard error of the mean $(\mathrm{n}=3)$. ${ }^{*} \mathrm{P}<0.05$ and ${ }^{* *} \mathrm{P}<0.01$ vs. control. IL, interleukin; PM, particulate matter; NW-PM2.5, non-water-soluble extracts; W-PM2.5, water-soluble extracts. 
A

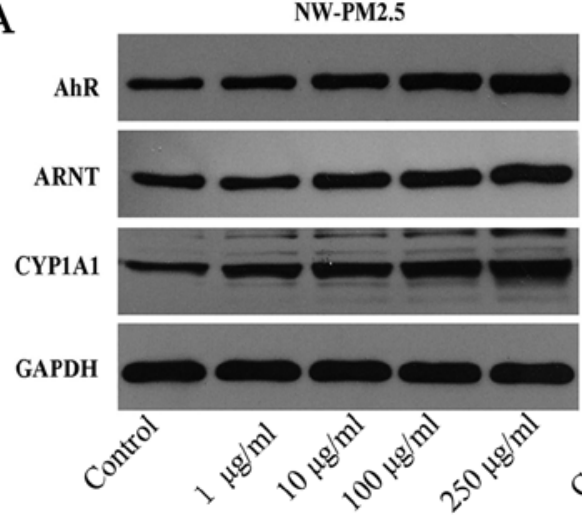

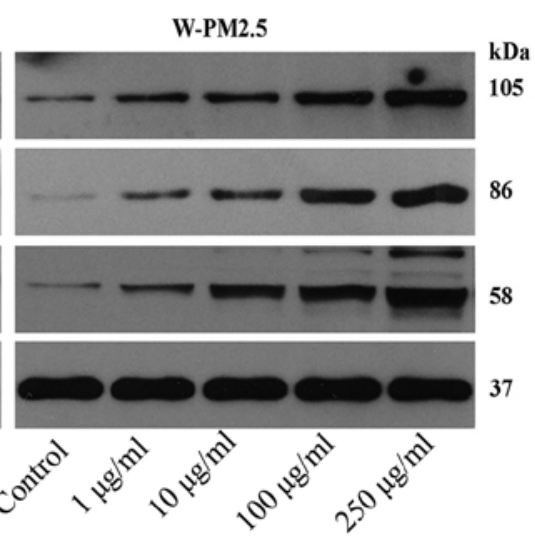
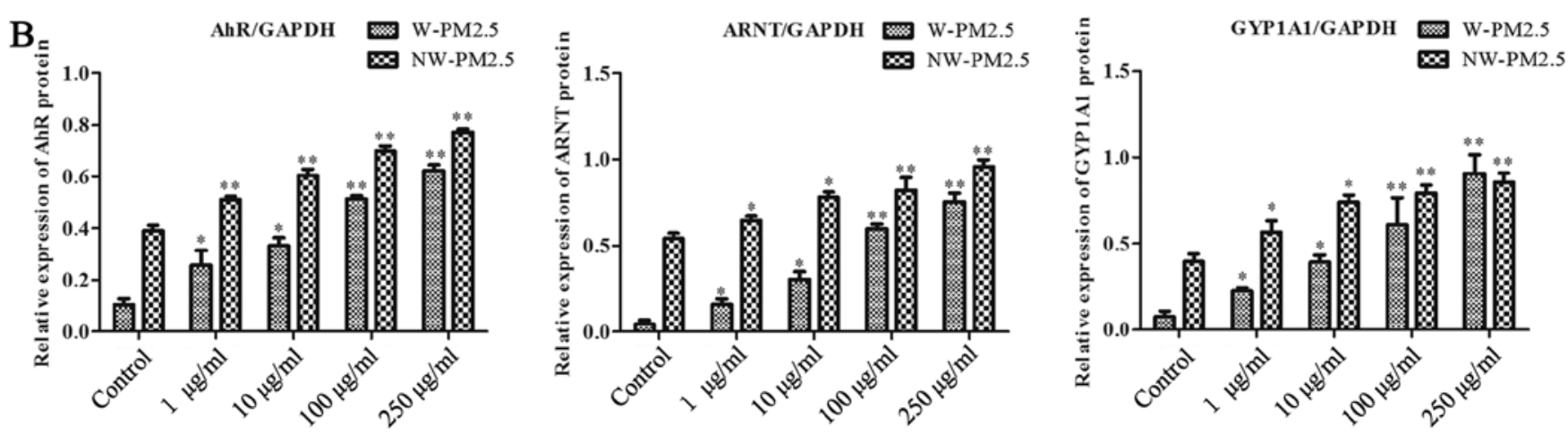

Figure 5. Western blot analysis of AhR, ARNT and CYP1A1 in PM2.5-exposed SZ95 sebocytes. Cell extracts were subjected to 10\% SDS-PAGE and western blot analysis with a primary antibody against AhR, ARNT and CYP1A1, respectively. GAPDH protein was used as an internal control. (A) Western blot analysis results of AhR, ARNT and CYP1A1. (B) Quantification of AhR, ARNT and CYP1A1 protein expression. " $\mathrm{P}<0.05$ and ${ }^{* *} \mathrm{P}<0.01$ vs. control. AhR, aryl hydrocarbon receptor; ARNT, AhR nuclear translocator protein; CYP1A1, cytoplasmic cytochrome P4501 A1; PM, particulate matter; NW-PM2.5, non-water-soluble extracts; W-PM2.5, water-soluble extracts; GAPDH, glyceraldehyde 3-phosphate dehydrogenase.

PM2.5 exposure regulated pro-inflammatory cytokine secretion in SZ95 sebocytes. Whether PM2.5 exposure regulated pro-inflammatory cytokine secretion was investigated by ELISA of pro-inflammatory cytokines (IL-1 $\alpha$, IL-6 and IL-8). NW-PM2.5 (Fig. 4A, C and E) and W-PM2.5 (Fig. 4B, D and F) exposure dose-dependently elevated the levels of all the evaluated pro-inflammatory cytokines that were released by SZ95 sebocytes, when compared with the unexposed control cells.

PM2.5 exposure activated AhR/CYPlA1 signaling in SZ95 sebocytes. In addition, whether AhR/CYP1A1 signaling is involved in the effect of PM2.5 exposure on SZ95 sebocytes was evaluated. Western blot analysis revealed that compared with unexposed control cells, SZ95 sebocyte exposure to NW-PM2.5 dose-dependently induced the elevation of protein expression levels of AhR, ARNT and CYP1A1. Similarly, the AhR, ARNT and CYP1A1 expression levels were reduced in the unexposed control cells, whereas the AhR, ARNT and CYP1A1 expression levels in SZ95 sebocytes were markedly increased in a dose-dependent manner by W-PM2.5 (Fig. 5).

Furthermore, immunocytochemical analysis (Fig. 6) demonstrated the localization of AhR, ARNT and CYP1A1 in PM2.5-exposed SZ95 sebocytes. Compared with unexposed control cells, significant AhR induction was observed in NW-PM2.5- (Fig. 6A) and W-PM2.5-exposed (Fig. 6B) cells. Similar to AhR, the staining for ARNT and CYP1A1 was signifi- cantly and dose-dependently increased in NW-PM2.5-exposed [ARNT (Fig. 6C); CYP1A1 (Fig. 6E)] and W-PM2.5-exposed [ARNT (Fig. 6D); CYP1A1 (Fig. 6F)] SZ95 sebocytes.

\section{Discussion}

The adverse effects of PM2.5 on respiratory diseases have been widely documented (24-26). However, the effects of PM2.5 on the function of human sebaceous glands have not been fully elucidated. To the best of our knowledge, this is the first study to investigate the influence of NW-PM2.5 and W-PM2.5 exposure on human sebocytes. The present study identified that PM2.5 exposure significantly inhibits the viability of SZ95 sebocytes, inducing cell toxicity. Furthermore, a high dose of NW-PM2.5 and W-PM2.5 exposure markedly reduced lipid synthesis in SZ95 sebocytes. In addition, pro-inflammatory cytokines, including IL-1 $\alpha$, IL-6 and IL-8, were demonstrated to be markedly elevated in PM2.5-exposed cells, and PM2.5 exposure induced $\mathrm{G}_{1}$ arrest in SZ95 sebocytes. Finally, the key AhR signaling pathway was activated following PM2.5 exposure in SZ95 sebocytes. A previous study demonstrated the cytotoxicity of PM2.5 on human HaCaT keratinocytes (11). Our study also identified that PM2.5 significantly inhibited cell viability, inducing toxicity on SZ95 sebocytes. The effects of PM2.5 exposure on cell cycle progression are controversial, as certain studies demonstrated that PM2.5 induced $G_{1}$ delay (27), while other studies found 

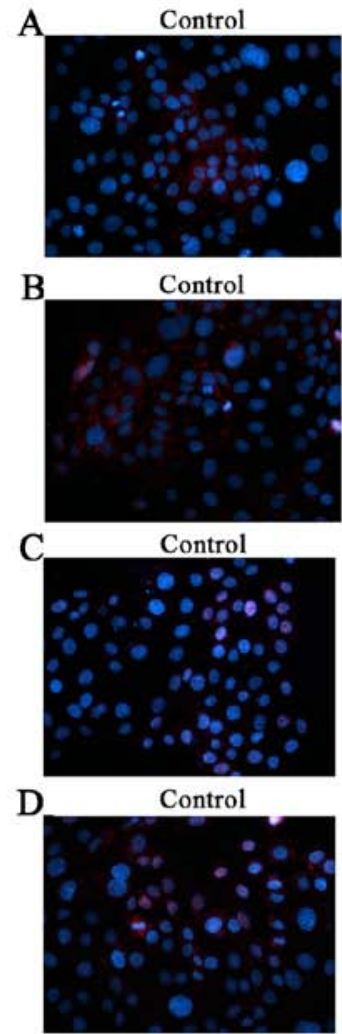

E

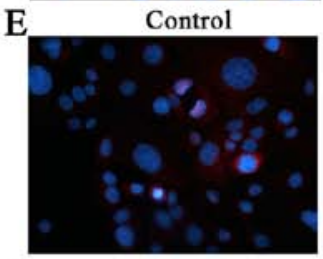

F

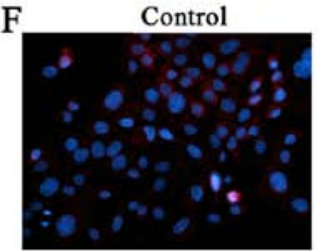

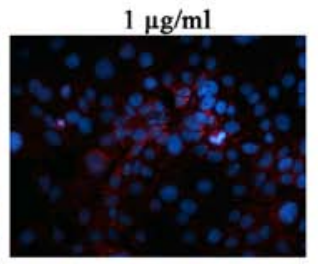

$1 \mu \mathrm{g} / \mathrm{ml}$

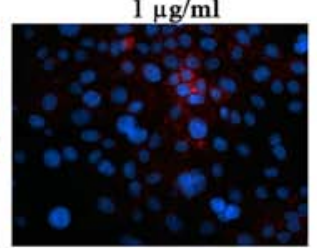

$1 \mu \mathrm{g} / \mathrm{ml}$
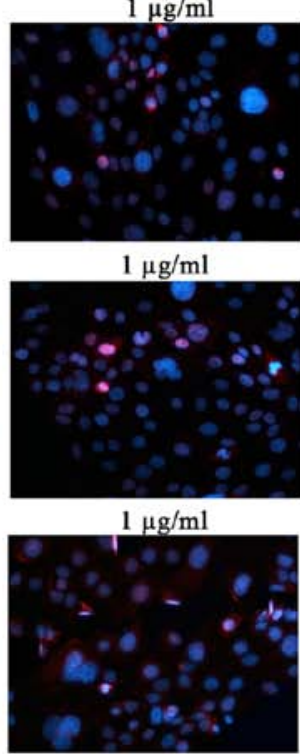

$1 \mu \mathrm{g} / \mathrm{ml}$

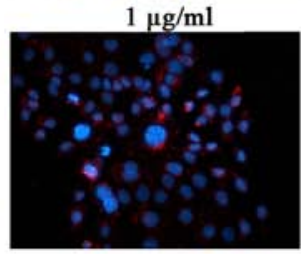

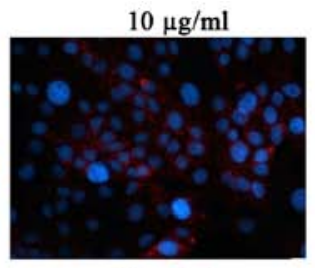

$10 \mu \mathrm{g} / \mathrm{ml}$
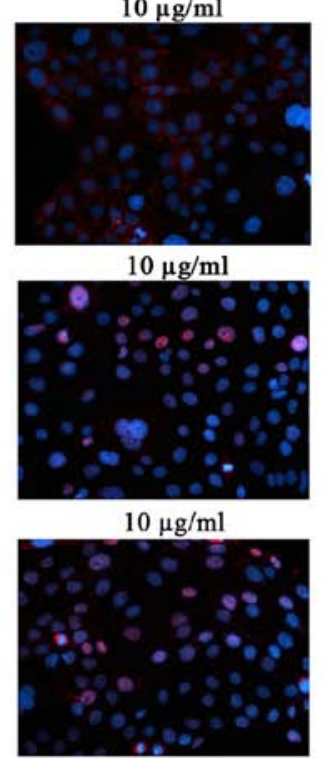

$10 \mu \mathrm{g} / \mathrm{ml}$

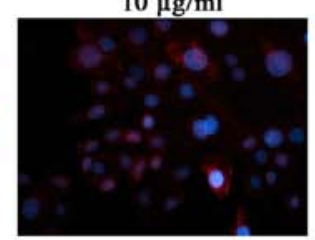

$10 \mu \mathrm{g} / \mathrm{ml}$

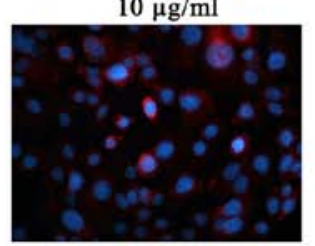

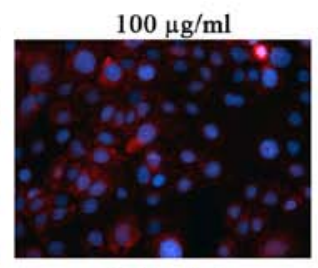

$100 \mu \mathrm{g} / \mathrm{ml}$
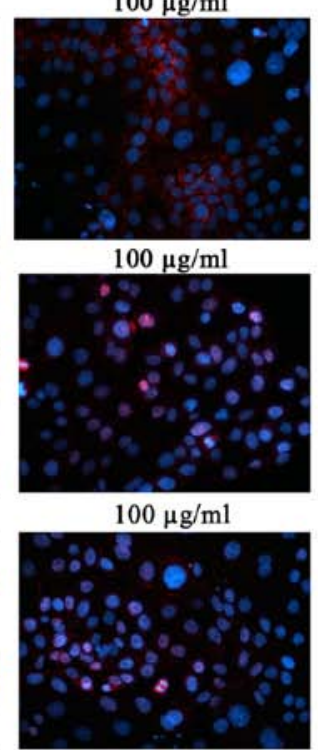

$100 \mu \mathrm{g} / \mathrm{ml}$

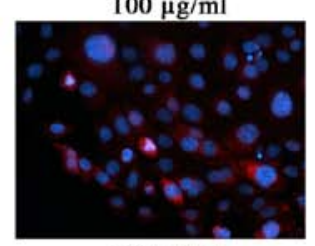

$100 \mu \mathrm{g} / \mathrm{ml}$

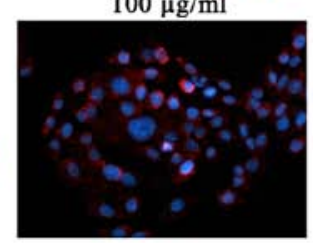

$250 \mu \mathrm{g} / \mathrm{ml}$

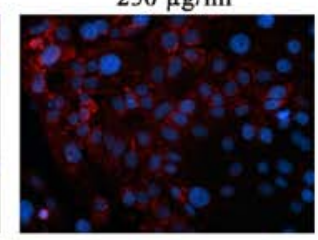

$250 \mu \mathrm{g} / \mathrm{ml}$

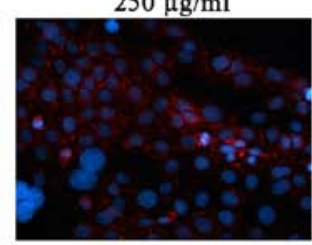

$250 \mu \mathrm{g} / \mathrm{ml}$

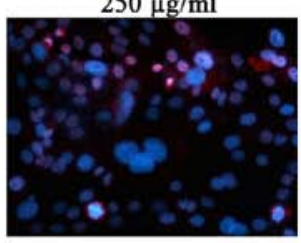

$250 \mu \mathrm{g} / \mathrm{ml}$

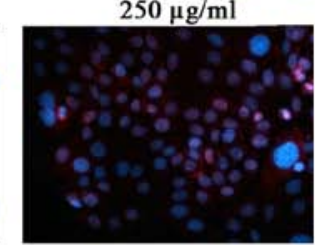

$250 \mu \mathrm{g} / \mathrm{ml}$

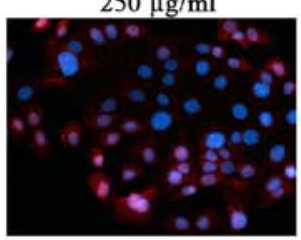

$250 \mu \mathrm{g} / \mathrm{ml}$

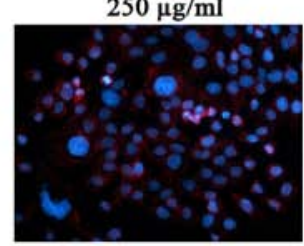

Figure 6. Immunofluorescent cytochemical analysis of AhR signaling following PM2.5 exposure in (A and B) AhR; (C and D) ARNT; (E and F) CYP1A1 in SZ95 sebocytes. (A, C and E) NW-PM2.5 exposure and (B, D and F) W-PM2.5 exposure (the magnification was x200). Nuclear staining was performed with DAPI. AhR, aryl hydrocarbon receptor; ARNT, AhR nuclear translocator protein; CYP1A1, cytochrome P4501 A1; PM, particulate matter; NW-PM2.5, non-water-soluble extracts; W-PM2.5, water-soluble extracts.

that PM2.5 arrested the cell cycle at the $\mathrm{G}_{2} / \mathrm{M}$ phase (28). In the present study, flow cytometry indicated that SZ95 sebocytes exposed to PM2.5 were arrested at the $G_{1}$ phase. As $G_{1}$ is the preparation phase of DNA synthesis, arresting the cell cycle at the $G_{1}$ phase directly inhibits cell growth. Therefore, cell proliferation suppression induced by PM2.5 is associated with $G_{1}$ arrest, and is consistent with the detected LDH increase.

Reduced lipid synthesis is associated with various diseases of the sebaceous gland, including chloracne $(29,30)$. In the present study, lipid synthesis markedly increased at a low PM2.5 concentration, while increased PM2.5 concentrations rapidly reduced the lipid synthesis in SZ95 sebocytes. In addition to activating lipid metabolism, SZ95 sebocytes were found to contribute to the inflammatory environment by stimulating the release of pro-inflammatory cytokines, such as IL- $1 \alpha$, IL-6 and IL-8. The results of the present study indicated that PM2.5 dose-dependently elevated the levels of IL-1 $\alpha$, IL-6 and IL-8, which are involved in the initiation of the pathogenesis of various types of sebaceous gland-associated disease $(31,32)$.

Previous studies have reported that PM2.5 (33) and extractable organic matter from PM2.5 $(34,35)$ may activate AhR in human cell lines. Consistent with these studies, the present study found that the protein expression level of AhR, ARNT and CYP1A1 was markedly enhanced in the PM2.5-exposed SZ95 sebocytes, indicating the activation of AhR signaling following PM2.5 exposure. Additionally, it has been demonstrated that AhR negatively regulates lipid synthesis in SZ95 sebocytes (16). Thus, the activated AhR signaling by PM2.5 exposure may lead to a reduction of lipid synthesis, which is consistent with the results of the present study. It is likely that PM2.5 exposure regulates the lipid synthesis in human SZ95 sebocytes by activating AhR/ARNT/CYP1A1 signaling. However, further studies are required to clarify the molecular mechanism involved. 
In conclusion, the present study investigated the influences of PM2.5 exposure on the functions of human SZ95 sebocytes. PM2.5 exposure was shown to exhibit inhibitory effects on cell proliferation, lipid synthesis, and stimulatory effects on inflammatory cytokine production and AhR signaling activation in human SZ95 sebocytes. These findings indicate that PM2.5 may increase the risk for the occurrence of sebaceous gland-associated diseases, which are dependent on inflammatory responses and lipid synthesis. While further studies are necessary to fully elucidate the molecular mechanism, the present study provides a foundation to better understand the complex interactions involved in extrinsic triggering of sebaceous gland-associated diseases.

\section{Acknowledgements}

The authors would like to thank Professor Qiang Ju, Dr Tingting $\mathrm{Hu}$ (Renji Hospital of Shanghai Jiao Tong University, Shanghai, China) and Dr Li Meng (Shanghai Dermatology Hospital, Shanghai, China) for their help in developing this project. The present study was supported by grants from CMA-L'OREAL China Skin/Hair Grant Funds (grant no. S2016131419) and the youth funds of Zhongnan Hospital of Wuhan University (grant no. 2015A014; Wuhan, China).

\section{References}

1. Kim KE, Cho D and Park HJ: Air pollution and skin diseases: Adverse effects of airborne particulate matter on various skin diseases. Life Sci 152: 126-134, 2016.

2. Krutmann J, Liu W, Li L, Pan X, Crawford M, Sore G and Seite S: Pollution and skin: From epidemiological and mechanistic studies to clinical implications. J Dermatol Sci 76: 163-168, 2014

3. Ju Q, Zouboulis CC and Xia L: Environmental pollution and acne: Chloracne. Dermatoendocrinol 1: 125-128, 2009.

4. Ju Q and Zouboulis CC: Endocrine-disrupting chemicals and skin manifestations. Rev Endocr Metab Disord 17: 449-457, 2016.

5. Ju Q, Fimmel S, Hinz N, Stahlmann R, Xia L and Zouboulis CC: 2,3,7,8-Tetrachlorodibenzo-p-dioxin alters sebaceous gland cell differentiation in vitro. Exp Dermatol 20: 320-325, 2011.

6. Hu T, Pan Z, Yu Q, Mo X, Song N, Yan M, Zouboulis CC, Xia L and Ju Q: Benzo(a)pyrene induces interleukin (IL)-6 production and reduces lipid synthesis in human SZ95 sebocytes via the aryl hydrocarbon receptor signaling pathway. Environ Toxicol Pharmacol 43: 54-60, 2016.

7. Ju Q, Yiang K, Zouboulis CC, Ring J and Chen W: Chloracne: From clinic to research. Zhonghua Pifuke Yixue Zazhi 30: 2-6, 2012.

8. Díaz RV and Rosa Dominguez E: Health risk by inhalation of PM2.5 in the metropolitan zone of the City of Mexico. Ecotoxicol Environ Saf 72: 866-871, 2009.

9. Dockery DW: Health effects of particulate air pollution. Ann Epidemiol 19: 257-263, 2009.

10. Weichenthal SA, Godri-Pollitt K and Villeneuve PJ: PM2.5, oxidant defence and cardiorespiratory health: A review. Environ Health 12: 40, 2013.

11. Li Q, Kang Z, Jiang S, Zhao J, Yan S, Xu F and Xu J: Effects of ambient fine particles PM2.5 on human HaCaT cells. Int J Environ Res Public Health 14: E72, 2017.

12. Sugihara K, Kitamura S, Yamada T, Ohta S, Yamashita K, Yasuda M and Fujii-Kuriyama Y: Aryl hydrocarbon receptor (AhR)-mediated induction of xanthine oxidase/xanthine dehydrogenase activity by 2,3,7,8-tetrachlorodibenzo-p-dioxin. Biochem Biophys Res Commun 281: 1093-1099, 2001.

13. Hong $\mathrm{CH}$, Lee $\mathrm{CH}$, Yu HS and Huang SK: Benzopyrene, a major polyaromatic hydrocarbon in smoke fume, mobilizes Langerhans cells and polarizes Th2/17 responses in epicutaneous protein sensitization through the aryl hydrocarbon receptor. Int Immunopharmacol 36: 111-117, 2016.

14. Jux B, Kadow S and Esser C: Langerhans cell maturation and contact hypersensitivity are impaired in aryl hydrocarbon receptor-null mice. J Immunol 182: 6709-6717, 2009.
15. Cho YC,Zheng W and Jefcoate CR: Disruption of cell-cell contact maximally but transiently activates AhR-mediated transcription in 10T1/2 fibroblasts. Toxicol Appl Pharmacol 199: 220-238, 2004.

16. Hu T, Wang D, Yu Q, Li L, Mo X, Pan Z, Zouboulis CC, Peng L, $\mathrm{Xia} \mathrm{L}$ and Ju Q: Aryl hydrocarbon receptor negatively regulates lipid synthesis and involves in cell differentiation of SZ95 sebocytes in vitro. Chem Biol Interact 258: 52-58, 2016.

17. Ju Q, Yu Q, Song N, Tan Y, Xia L and Zouboulis CC: Expression of aryl hydrocarbon receptor in human epidermis, hair follicles and sebaceous glands and its significance. Zhonghua Pifuke Zazhi 44: 761-764, 2011.

18. Esser C, Bargen I, Weighardt H, Haarmann-Stemmann T and Krutmann J: Functions of the aryl hydrocarbon receptor in the skin. Semin Immunopathol 35: 677-691, 2013.

19. Yu Q, Hu T, Mo X, Zhang C, Xia L, Zouboulis CC and Ju Q: Effect of tetrachlorodibenzo-p-dioxin on the expression of cytochrome P4501A1 in human SZ95 sebocytes and its significance. Zhonghua Pifuke Zazhi 46: 557-560, 2013.

20. Kalmes M, Hennen J, Clemens J and Blömeke B: Impact of aryl hydrocarbon receptor (AhR) knockdown on cell cycle progression in human HaCaT keratinocytes. Biol Chem 392: 643-651, 2011.

21. Tauchi M, Hida A, Negishi T, Katsuoka F, Noda S, Mimura J, Hosoya T, Yanaka A, Aburatani H, Fujii-Kuriyama Y, et al: Constitutive expression of aryl hydrocarbon receptor in keratinocytes causes inflammatory skin lesions. Mol Cell Biol 25: 9360-9368, 2005.

22. Kakimoto K, Nagayoshi H, Konishi Y, Kajimura K, Ohura T, Nakano T, Hata M, Furuuchi M, Tang N, Hayakawa K, et al: Size distribution of chlorinated polycyclic aromatic hydrocarbons in atmospheric particles. Arch Environ Contam Toxicol 72: 58-64, 2017.

23. Jeong SC, Song MK, Cho Y, Lee E and Ryu JC: Integrative analysis of mRNA and microRNA expression of a human alveolar epithelial cell(A549) exposed to water and organic-soluble extract from particulate matter (PM)2.5. Environ Toxicol 32: 302-310, 2017.

24. Yang B, Chen D, Zhao H and Xiao C: The effects for PM2.5 exposure on non-small-cell lung cancer induced motility and proliferation. Springerplus 5: 2059, 2016.

25. Zhou Z, Liu Y, Duan F, Qin M, Wu F, Sheng W, Yang L, Liu J and $\mathrm{He} \mathrm{K}$ : Transcriptomic analyses of the biological effects of airborne PM2.5 exposure on human bronchial epithelial cells. PLoS One 10: e0138267, 2015.

26. Huang Q, Zhang J, Peng S, Tian M, Chen J and Shen H: Effects of water soluble PM2.5 extracts exposure on human lung epithelial cells (A549): A proteomic study. J Appl Toxicol 34: 675-687, 2014.

27. Zhang J, Ghio AJ, Gao M, Wei K, Rosen GD and Upadhyay D: Ambient particulate matter induces alveolar epithelial cell cycle arrest: Role of $\mathrm{G}_{1}$ cyclins. FEBS Lett 581: 5315-5320, 2007.

28. Longhin E, Holme JA, Gutzkow KB, Arlt VM, Kucab JE, Camatini $\mathrm{M}$ and Gualtieri M: Cell cycle alterations induced by urban PM2.5 in bronchial epithelial cells: Characterization of the process and possible mechanisms involved. Part Fibre Toxicol 10: 63, 2013 .

29. Chen W, Obermayer-Pietsch B, Hong J-B, Melnik BC, Yamasaki O, Dessinioti C, Ju Q, Liakou AI, Al-Khuzaei S, Katsambas A, et al: Acne-associated syndromes: Models for better understanding of acne pathogenesis. J Eur Acad Dermatol Venereol 25: 637-646, 2011.

30. Zouboulis CC, Picardo M, Ju Q, Kurokawa I, Törőcsik D, Bíró T and Schneider MR: Beyond acne: Current aspects of sebaceous gland biology and function. Rev Endocr Metab Disord 17: 319-334, 2016.

31. Zouboulis CC, Jourdan E and Picardo M: Acne is an inflammatory disease and alterations of sebum composition initiate acne lesions. J Eur Acad Dermatol Venereol 28: 527-532, 2014.

32. Ganceviciene R, Graziene V, Fimmel S and Zouboulis CC: Involvement of the corticotropin-releasing hormone system in the pathogenesis of acne vulgaris. Br J Dermatol 160: 345-352, 2009.

33. Zhang H, Yao Y, Chen Y, Yue C, Chen J, Tong J, Jiang Y and Chen T: Crosstalk between AhR and wnt/ $\beta$-catenin signal pathways in the cardiac developmental toxicity of PM2.5 in zebrafish embryos. Toxicology 355-356: 31-38, 2016.

34. Borgie M, Ledoux F, Verdin A, Cazier F, Greige H, Shirali P, Courcot D and Dagher Z: Genotoxic and epigenotoxic effects of fine particulate matter from rural and urban sites in Lebanon on human bronchial epithelial cells. Environ Res 136: 352-362, 2015.

35. Líbalová H, Krčková S, Uhlířová K, Milcová A, Schmuczerová J, Ciganek M, Kléma J, Machala M, Šrám RJ and Topinka J: Genotoxicity but not the AhR-mediated activity of PAHs is inhibited by other components of complex mixtures of ambient air pollutants. Toxicol Lett 225: 350-357, 2014. 